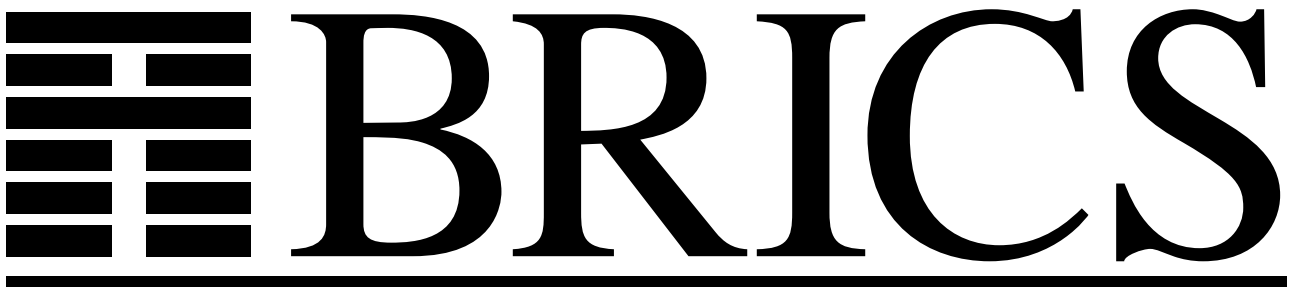

Basic Research in Computer Science

\title{
New Algorithms for Exact Satisfiability
}

Jesper Makholm Byskov

Bolette Ammitzbøll Madsen

Bjarke Skjernaa 
Copyright (c) 2003, Jesper Makholm Byskov \& Bolette Ammitzbøll Madsen \& Bjarke Skjernaa.

BRICS, Department of Computer Science University of Aarhus. All rights reserved.

Reproduction of all or part of this work is permitted for educational or research use on condition that this copyright notice is included in any copy.

See back inner page for a list of recent BRICS Report Series publications. Copies may be obtained by contacting:

\author{
BRICS \\ Department of Computer Science \\ University of Aarhus \\ Ny Munkegade, building 540 \\ DK-8000 Aarhus C \\ Denmark \\ Telephone: +4589423360 \\ Telefax: $\quad+4589423255$ \\ Internet: BRICS@brics.dk
}

BRICS publications are in general accessible through the World Wide Web and anonymous FTP through these URLs:

http: //www.brics.dk

ftp: / / ftp.brics.dk

This document in subdirectory RS/03/30/ 


\title{
New Algorithms for Exact Satisfiability*
}

\author{
Jesper Makholm Byskov ${ }^{\dagger}$ Bolette Ammitzbøll Madsen \\ Bjarke Skjernaa ${ }^{\ddagger}$ \\ BRICS $\$$ Department of Computer Science \\ University of Aarhus, Denmark \\ \{jespermn, bolette, skjernaa\}@brics. dk
}

8th October 2003

\begin{abstract}
The Exact Satisfiability problem is to determine if a CNFformula has a truth assignment satisfying exactly one literal in each clause; Exact 3-Satisfiability is the version in which each clause contains at most three literals. In this paper, we present algorithms for Exact Satisfiability and Exact 3-Satisfiability running in time $O\left(2^{0.2325 n}\right)$ and $O\left(2^{0.1379 n}\right)$, respectively. The previously best algorithms have running times $O\left(2^{0.2441 n}\right)$ for Exact Satisfiability (Monien, Speckenmeyer and Vornberger (1981)) and $O\left(2^{0.1626 n}\right)$ for Exact 3-Satisfiability (Kulikov and independently Porschen, Randerath and Speckenmeyer (2002)). We extend the case analyses of these papers and observe, that a formula not satisfying any of our cases has a small number of variables, for which we can try all possible truth assignments and for each such assignment solve the remaining part of the formula in polynomial time.
\end{abstract}

* Partially supported by the Future and Emerging Technologies programme of the EU under contract number IST-1999-14186 (ALCOM-FT).

${ }^{\dagger}$ Part of this research was done while visiting School of Information \& Computer Science, University of California, Irvine.

${ }^{\ddagger}$ Part of this research was done while visiting Max-Planck-Institut für Informatik, Saarbrücken, Germany.

$\S$ Basic Research in Computer Science (www.brics.dk), funded by the Danish National Research Foundation. 


\section{Introduction}

The ExACT SATISFIABILITY (XSAT) problem is a variant of SATISFIABILITY (SAT), where the difference is that in XSAT a clause is satisfied if exactly one of its literals is true. ExACT 3-SATISFIABILITY (X3SAT) ${ }^{1}$ is the variant of XSAT in which each clause contains at most three literals. XSAT is NP-complete even when restricted to clauses containing at most three literals and all variables occurring only unnegated [8]. XSAT with all variables occurring at most twice can be solved in polynomial time $[5]^{2}$

XSAT can easily be solved in time $O\left(2^{n}\right)$ (we will ignore polynomial factors when stating running times, since these are all exponential) by enumerating all possible truth assignments to the $n$ variables. In 1981, Schroeppel and Shamir were the first to give a faster algorithm. Their algorithm solves a class of problems of which XSAT and KNAPSACK are the most prominent in time $O\left(2^{n / 2}\right)$ and space $O\left(2^{n / 4}\right)[9]$. The same year, Monien, Speckenmeyer and Vornberger [5] gave an algorithm solving only XSAT, but in time $O\left(2^{0.2441 n}\right)^{3}$ using only polynomial space. This is the previously best algorithm for XSAT.

X3SAT can of course be solved by an algorithm solving XSAT, but in recent years faster algorithms for X3SAT have been designed. The first was by Drori and Peleg [1] and runs in time $O\left(2^{0.2072 n}\right)$; several improvements followed and the previously best running time for X3SAT is $O\left(2^{0.1626 n}\right)$ and was achieved independently by Kulikov [2] and Porschen, Randerath and Speckenmeyer [6] $]^{4}$ in 2002.

Except for the algorithm by Schroeppel and Shamir [9], all the algorithms above are branch-and-reduce algorithms. A branch-and-reduce algorithm branches by making recursive calls on smaller formulas, such that the original formula is satisfiable if and only if at least one of the smaller formulas are satisfiable. In each branch, the algorithm reduces the formula by replacing it with another formula that is satisfiable if and only if the original formula is and that contains fewer variables. Fast branch-and-reduce algorithms rely on good decisions about what to

\footnotetext{
${ }^{1}$ EXACT 3-SAtisfiability is also called One-In-Three SATiSfiability.

${ }^{2}$ They state that a generalised version of XSAT with variables occurring at most twice, called $\operatorname{MAX}(\{\leq,=, \geq\}, \cdot, 2)$, reduces to Perfect Matching. The proof is in the technical report [4].

${ }^{3}$ Note that [5] only states the time $O\left(2^{n / 4}\right)$. The time complexity of $O\left(2^{0.2441 n}\right)$ is proved in the technical report [4].

${ }^{4}$ See Porschen, Randerath and Speckenmeyer [7] for an extended abstract.
} 
branch on and good reduction rules.

In this paper, we present new branch-and-reduce algorithms for XSAT and X3SAT running in time $O\left(2^{0.2325 n}\right)$ and $O\left(2^{0.1379 n}\right)$, respectively. We introduce new reductions for both XSAT and X3SAT and improve the case analyses by a more careful analysis of the worst cases, which for some cases involves splitting them into more cases. Our main improvement, however, lies in our handling of sparse formulas: if the number of variables occurring at least three times in the formula is small, we can enumerate all possible truth assignments to these variables. For each assignment, the remaining formula contains only variables occurring at most twice, so we can decide in polynomial time, if it is satisfiable [5].

\section{Preliminaries}

\subsection{Definitions}

We are given a set of variables, which we will denote by using the letters $x, y, z, w$ and $u$. A literal is either a variable $x$ or the negation of a variable $\bar{x}$; we use $\tilde{x}$ to denote a literal that is either $x$ or $\bar{x}$. A clause is a collection of literals, written as $\left(\tilde{x}_{1}, \ldots, \tilde{x}_{l}\right)$; we use the letter $C$ to denote clauses. Sometimes, we will think of a clause as a set of literals (actually a multiset, since a clause can contain more than one of each literal); we use $(\tilde{x}, C)$ to denote a clause with $\tilde{x}$ and all the literals in $C$. A formula is a set of clauses usually written as $C_{1} \wedge C_{2} \wedge \cdots \wedge C_{m}$; we use the letter $F$ to denote formulas. In intermediate steps of our algorithm we allow clauses to contain constants (true or false). The size of a formula is the number of literals and constants contained in the formula.

$\mathrm{XSAT}$ is the problem of given a formula $F$ with $m$ clauses over $n$ variables to decide, if there exists an assignment to the variables, such that exactly one literal in each clause is true.

We let $V(F)$ denote the variables occurring in $F$. An $(a, b)$-occurrence is a variable occurring $a$ times unnegated and $b$ times negated or vice versa in $F$; a unique variable is a variable occurring only once. We will assume for simplicity, that when we look at a variable the first occurrence is unnegated.

We let $F[x \leftarrow y]$ where $y$ is either a constant or a literal denote $F$ with $x$ replaced by $y$ and $\bar{x}$ replaced by $\bar{y}$; similarly, we let $F[C \leftarrow$ false $]$ denote $F$ with all literals in $C$ replaced by false and their negations by true. 
In X3SAT, a cycle is a list of clauses $\left(y_{1}, \tilde{z}_{1}, z_{2}\right),\left(y_{2}, \tilde{z}_{2}, z_{3}\right), \ldots$, $\left(y_{k}, \tilde{z}_{k}, z_{1}\right)$ where neighbour clauses and the first and last clause share a variable and the $z_{i}$ 's are different variables.

\subsection{Branching}

Our algorithms make recursive calls on formulas with fewer variables. If $C$ is a clause in the formula and $C^{\prime} \subsetneq C$ then in a satisfying assignment for the formula either all literals in $C^{\prime}$ are false or exactly one is true. We use three different types of branches: branching on $C^{\prime}$ meaning that the recursive calls are on $C^{\prime} \wedge F$ (in this case the formula can be reduced immediately afterwards, such that there will be fewer variables and clauses) and $F\left[C^{\prime} \leftarrow\right.$ false]; we will denote the first branch "setting $C^{\prime}$ to true" and the second "setting $C^{\prime}$ to false". We can also branch on two variables $x_{1}, x_{2}$ meaning that the recursive calls are on $F\left[x_{1} \leftarrow\right.$ true $]$, $F\left[x_{2} \leftarrow\right.$ true $]$ and $F\left[x_{1}, x_{2} \leftarrow\right.$ false $]$. Finally, we can branch on $x_{1}$; the two branches are then $F\left[x_{1} \leftarrow\right.$ true $]$ and $F\left[x_{1} \leftarrow\right.$ false $]$.

\subsubsection{Sparse formulas}

We call a formula $k$-sparse, if the number of variables occurring at least three times is at most $n / k$. To decide if a $k$-sparse formula is satisfiable, we enumerate all possible truth assignments to these $n / k$ variables; for each assignment, all variables in the remaining part of the formula occur at most twice, so we can decide in polynomial time, if it is satisfiable. The total running time is $O\left(2^{n / k}\right)$. We use this for XSAT with $k=5$ and for X3SAT with $k=\frac{15}{2}$.

\subsection{Branching vectors}

In each branch of the algorithm, we remove a certain number of variables using the reductions; then we get a recursion for the running time of the form $T(n)=T\left(n-t_{1}\right)+T\left(n-t_{2}\right)+\cdots+T\left(n-t_{k}\right)$. We call $t=\left(t_{1}, t_{2}, \ldots, t_{k}\right)$ the branching vector of this branch. The solution to the recursion is $T(n)=\alpha_{t}^{n}$, where $\alpha_{t}$ is the positive root of $1-\frac{1}{x^{t_{1}}}-\frac{1}{x^{t_{2}}}-$ $\cdots-\frac{1}{x^{t} k}$; we call $\alpha_{t}$ the value of $t$ and the value of a branching vector is decreasing as a function of the entries in the vector. Proofs of these results can be found in a manuscript by Kullmann and Luckhardt [3]. The logarithms of the values of all branching vectors occurring in this paper are either stated in Table 1 or are smaller than one of them by 
Table 1: Branching vectors and the logarithms of their values (rounded)

(a) XSAT

\begin{tabular}{|rc|rc|}
\hline \multicolumn{1}{|c|}{$t$} & $\log _{2}\left(\alpha_{t}\right)$ & \multicolumn{1}{c|}{$t$} & $\log _{2}\left(\alpha_{t}\right)$ \\
\hline$(12,1)$ & 0.2302 & $(11,11,3)$ & 0.2216 \\
$(8,2)$ & 0.2325 & $(10,8,4)$ & 0.2325 \\
$(6,3)$ & 0.2315 & $(13,7,4)$ & 0.2258 \\
$(5,4)$ & 0.2232 & & \\
\hline
\end{tabular}

(b) X3SAT

\begin{tabular}{|rc|}
\hline \multicolumn{1}{|c|}{$t$} & $\log _{2}\left(\alpha_{t}\right)$ \\
\hline$(12,4)$ & 0.1379 \\
$(11,5)$ & 0.1317 \\
$(9,6)$ & 0.1353 \\
$(8,7)$ & 0.1336 \\
\hline
\end{tabular}

monotonicity. The running time of the whole algorithm is $O\left(2^{\log _{2} \alpha \cdot n}\right)$, where $\alpha$ is the largest of the $\alpha_{t}$ 's.

\section{The algorithms}

Both our algorithms have the following structure: first, the algorithm reduces the formula using the reductions from Section 3.1. If the reduced formula (we call a formula reduced, if none of the reductions from Section 3.1 is applicable) contains no clauses it is satisfied and if it contains the empty clause it can not be satisfied. If the formula only contains variables that occur at most twice in the formula, the algorithm decides in polynomial time if the formula is satisfiable; otherwise, the algorithm branches depending on whether the formula contains certain subformulas. The algorithm branches on the first matching case, which means that when it is in one case, no part of the formula matches any previous cases. The cases are described for XSAT in Section 3.2 and for X3SAT in Section 3.3. For simplicity, we ignore symmetries when this does not lead to confusion, so if we have two variables $y_{1}$ and $y_{2}$ or two clauses $C_{1}$ and $C_{2}$ that occur symmetrically and one of them has a certain property, we will just assume it is either.

\subsection{Reductions}

In this section, we present the reductions that are used in our algorithms; first, we present some common reductions used in both algorithms and then specific ones for the two algorithms. The reduction procedure for XSAT uses reductions (1) to (13) and the reduction procedure for X3SAT uses (1) to (8) and (14) and (15). The reductions are applied repeatedly top-down until no reduction applies. If any of the reductions 
either assign a variable both true and false or a constant is assigned its opposite value, the reduction procedures replace the whole formula with the empty clause.

When we branch, we call the algorithms recursively on smaller formulas. We show how to apply specific reductions to remove the stated number of variables. One can also show that applying the reductions top-down leads to the same or better branching vectors.

\subsubsection{Common reductions}

The common reductions are standard reductions also used by, e.g., Kulikov [2]. $F$ denotes the entire left hand side of the reduction.

$$
\begin{aligned}
(\text { true }, C) & \wedge F^{\prime} \rightarrow F^{\prime}[C \leftarrow \text { false }] \\
(\text { false }, C) & \wedge F^{\prime} \rightarrow C \wedge F^{\prime} \\
(x) & \wedge F^{\prime} \rightarrow F^{\prime}[x \leftarrow \text { true }] \\
(x, y) & \wedge F^{\prime} \rightarrow F^{\prime}[y \leftarrow \bar{x}] \\
(x, x, C) & \wedge F^{\prime} \rightarrow F[x \leftarrow \text { false }] \\
(x, \bar{x}, C) & \wedge F^{\prime} \rightarrow F^{\prime}[C \leftarrow \text { false }] \\
(x, y, C) \wedge\left(x, \bar{y}, C^{\prime}\right) & \wedge F^{\prime} \rightarrow F[x \leftarrow \text { false }] \\
(x, y, C) \wedge\left(\bar{x}, \bar{y}, C^{\prime}\right) & \wedge F^{\prime} \rightarrow F[y \leftarrow \bar{x}]
\end{aligned}
$$

\subsubsection{Reductions for XSAT}

Reduction (9) removes variables $x$ that only occur unnegated and only in clauses with a unique variable or with literal $y$ (and $y$ is in no clauses without $x$ ). Note, that either $k$ or $l$ can be zero.

$$
\begin{array}{rr}
\left(x, y, C_{1}\right) \wedge \cdots \wedge\left(x, y, C_{k}\right) \wedge\left(x, u_{1}, C_{1}^{\prime}\right) \wedge \cdots \wedge\left(x, u_{l}, C_{l}^{\prime}\right) \wedge F^{\prime} \rightarrow \\
x, y \notin V\left(F^{\prime}\right), u_{i} \text { unique } & F[x \leftarrow \text { false }]
\end{array}
$$

Reduction (9) is not used for X3SAT, as (14) or (15) can be used instead.

Reductions (10) and (11) are called resolution; resolution is a wellknown technique for removing variables occurring both unnegated and negated and can also be used for solving SAT formulas. The idea is, that we can remove a variable $x$ occurring both unnegated and negated and make all possible combinations of the clauses that contained $x$ and the 
clauses that contained $\bar{x}$. If $x$ is an $(a, b)$-occurrence, this will replace $a+b$ clauses with $a b$ clauses, so we only use it for $(a, 1)$ - and $(2,2)$-occurrences.

$$
\begin{gathered}
(\bar{x}, C) \wedge \underset{x \notin V\left(F^{\prime}\right)}{\left(x, C_{1}\right) \wedge}\left(x, C_{k}\right) \wedge F^{\prime} \rightarrow \\
\left(C, C_{1}\right) \wedge \cdots \wedge\left(C, C_{k}\right) \wedge F^{\prime} \\
\left(\bar{x}, C_{1}\right) \wedge\left(\bar{x}, C_{2}\right) \wedge\left(\underset{x \notin}{\left(x, F_{1}^{\prime}\right)} \wedge\left(x, C_{2}^{\prime}\right) \wedge F^{\prime} \rightarrow\right. \\
\left(C_{1}, C_{1}^{\prime}\right) \wedge\left(C_{1}, C_{2}^{\prime}\right) \wedge\left(C_{2}, C_{1}^{\prime}\right) \wedge\left(C_{2}, C_{2}^{\prime}\right) \wedge F^{\prime}
\end{gathered}
$$

Resolution is not used for X3SAT, as it creates clauses with more than three variables.

Reduction (12) removes clauses that have another clause as subset, and (13) reduces the formula if a clause shares all but one variable with another.

$$
\begin{array}{r}
C \wedge \underset{C \subseteq C^{\prime}}{C^{\prime} \wedge F^{\prime}} \rightarrow C \wedge F^{\prime}\left[C^{\prime} \backslash C \leftarrow \text { false }\right] \\
\left(x, C^{\prime}\right) \wedge\left(C, C^{\prime}\right) \wedge F^{\prime} \rightarrow\left(x, C^{\prime}\right) \wedge(\bar{x}, C) \wedge F^{\prime}
\end{array}
$$

Reduction (12) and (13) are not used for X3SAT as (14) handles the same cases when the clauses have size three.

Lemma 1. In a reduced XSAT formula, for all pairs of clauses, each has at least two variables that do not occur in the other.

Proof. All clauses contain at least three literals by (3) and (4), so we only need to consider clauses having at least two variables in common. Common variables must occur the same (unnegated or negated) by (7) and (8). No clause is a subset of another by (12), and for any pair of clauses both have at least two literals that do not occur in the other by 13 , so the lemma is true.

\subsubsection{Reductions for X3SAT}

Reduction (14) is also a standard reduction, but we only use it for X3SAT. Reduction (15) reduces formulas containing two variables that only occur unnegated and only in clauses with a unique variable, except for one clause, where one occurs unnegated and the other negated. Note, that both variables can be unique themselves.

$$
\begin{array}{cl}
\left(x, y, z_{1}\right) \wedge\left(x, y, z_{2}\right) \wedge F^{\prime} & \rightarrow\left(x, y, z_{1}\right) \wedge F^{\prime}\left[z_{2} \leftarrow z_{1}\right] \\
\left(\bar{x}_{1}, x_{2}, y\right) \wedge F^{\prime} & \rightarrow F\left[x_{2} \leftarrow \text { false }\right] \\
x_{1} \text { and } x_{2} \text { only occur unnegated and } \\
\text { in clauses with a unique variable in } F^{\prime}
\end{array}
$$


Remark. A reduced X3SAT formula contains no constants or 1- or 2clauses by (1) to (4) and no two clauses have more than one variable in common by (7), (8) and (14); also, no clause has more than one unique variable and all $(a, 0)$ - and $(a, 1)$-occurrences that are not unique are in a clause with no unique variables by (15).

\subsubsection{Soundness and complexity}

The following lemma states that the reductions are sound, that is, the reduced formula is satisfiable if and only if the original formula is.

Lemma 2. Reductions (1) to (15) are sound.

Proof. To prove that (1) to (8) and (10) to (14) are sound we just note, that exactly one literal from a clause must be true and exactly one of $x$ and $\bar{x}$ must be true.

In (9), $x$ can be assumed to be false, as a satisfying assignment with $x$ true can be changed to a satisfying assignment with $x$ false by making $y$ and the unique variables true instead. Similarly in (15), a satisfying assignment with $x_{2}$ true must have $x_{1}$ true, and it can be changed by setting $x_{1}$ and $x_{2}$ false and all the unique variables true.

The next two lemmas show, that during the reduction procedures the size of the formula is never larger than the maximum of the size of the original formula and $2 m n$. We use this to show, that the reduction procedures run in polynomial time in the size of the formula.

Lemma 3. When the reduction procedures run on a formula $F$ with $m$ clauses and $n$ variables the intermediate formulas are never larger than $\max (|F|, 2 m n)$.

Proof. For X3SAT it is obvious that the size of the formula is never larger than $\max (|F|, 3 m)$.

For XSAT, none of the reductions increases the number of variables or clauses in the formula. The reduction procedure first applies reductions (1) to (6), which all decrease the size of the formula. After it has run, the formula contains no constants and no variable occurs more than once in the same clause; thus, the size of the formula is at most $\mathrm{mn}$.

The only reductions which can make the formula larger are resolution (10) and (11)), but the number of literals in a clause after resolution is still bounded by $2 n$ and will be reduced to $n$ before we perform resolution again; thus, after the first applications of (1) to (6), the size of the formula is always bounded by $2 m n$. 
Lemma 4. The reduction procedures run in polynomial time in the size of the formula.

Proof. For each reduction, the algorithm can in polynomial time in the size of the formula check whether it is applicable and if so apply it.

Resolution (10) and (11) ) removes a variable from the formula, so they can be applied at most $n$ times, since no reduction add variables. All the other reductions reduce the size of the formula (for (13) just note that $C^{\prime}$ has size at least two by (4)). Since the size of the formula is at most $\max (|F|, 2 m n)$, the reduction procedures run in polynomial time in $|F|$.

\subsection{The algorithm for XSAT}

In this section, we present our algorithm for XSAT and show that it achieves a branching vector of $(8,2)$ corresponding to a running time of $O\left(2^{0.2325 n}\right)$. The previously best algorithm is by Monien et al. [5] and has worst case branching vector $(11,1)$ corresponding to a running time of $O\left(2^{0.2441 n}\right)$.

\subsubsection{Variables occurring both unnegated and negated}

If the formula $F$ contains a variable occurring both unnegated and negated, it must occur at least three times unnegated and twice negated or vice versa; otherwise, it would have been removed by resolution ( 10$)$ or (11)). Let $x$ be the corresponding literal occurring at least three times unnegated and twice negated as in the clauses in Figure 1. The

$$
\begin{array}{lll}
\left(x, C_{1}\right) & \left(\bar{x}, C_{1}^{\prime}\right) & x=\text { true }: C_{1}=C_{2}=C_{3}=\text { false } \\
\left(x, C_{2}\right) & \left(\bar{x}, C_{2}^{\prime}\right) & x=\text { false }: C_{1}^{\prime}=C_{2}^{\prime}=\text { false } \\
\left(x, C_{3}\right) &
\end{array}
$$

\footnotetext{
Figure 1: Branching on at least a $(3,2)$-occurrence $x$.
}

algorithm branches on $x$. By Lemma 1 and a simple counting argument, $C_{1}, C_{2}$ and $C_{3}$ must contain at least six different variables in total and $C_{1}^{\prime}$ and $C_{2}^{\prime}$ at least four; thus, branching on $x$ yields a branching vector of at least $(7,5)$. 


\subsubsection{Two clauses having at least two variables in common}

Suppose $F$ contains two clauses having more than one variable in common as in Figure 2, with $C_{1}$ and $C_{2}$ not having any variables in common and $|C| \geq 2$. By Lemma 1, also $\left|C_{1}\right|,\left|C_{2}\right| \geq 2$. If the two clauses are not

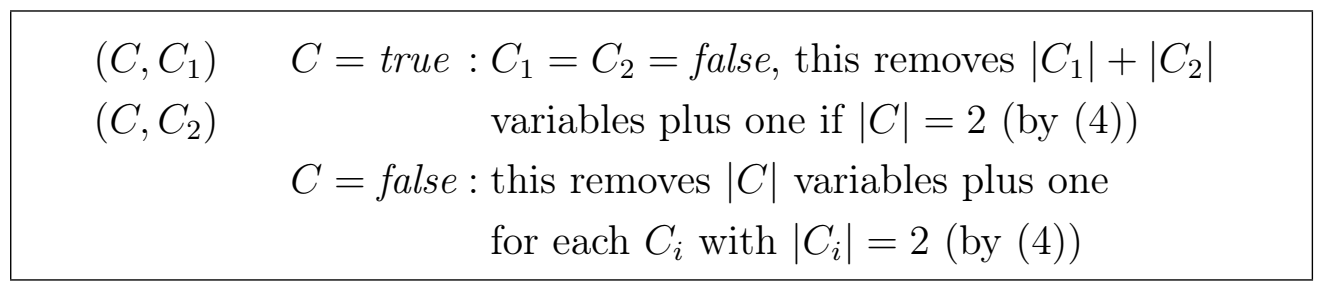

Figure 2: Two clauses having at least two variables in common.

two 5-clauses having exactly two variables in common, the algorithm branches on $C$ as shown in the figure. Since $C, C_{1}$ and $C_{2}$ all have size at least two, this removes at least four variables when $C$ is set to true and two when $C$ is set to false. Now, if any of the clauses are 2-clauses this removes one extra variable in one branch and if they are at least 3 -clauses we remove at least one more in the other branch. All in all we get branching vectors of at least $(5,4),(6,3)$ or $(7,2)$, but $(7,2)$ only if we had two 5-clauses having two variables in common. Having excluded that case, which we deal with later, we have $(8,2)$ as the worst case.

\subsubsection{Variables occurring at least four times}

If $F$ contains a variable $x$ occurring at least four times and either with at least eleven different variables or in a 3-clause, the algorithm branches on $x$. If it occurs with eleven different variables, this yields a branching vector of at least $(12,1)$ and if $x$ is in a 3 -clause, we get a branching vector of at least $(9,2)$, since $x$ must occur with at least eight different variables by Lemma 1 .

If $F$ contains a variable $x$ occurring at least four times that does not satisfy the previous case, we pick four of the clauses containing $x$; since the only clauses having more than one variable in common are 5-clauses having two variables in common, these must be 5-clauses pairwise having two variables in common as in Figure 3. Then the algorithm branches on $\left(x, y_{1}\right)$. When it is set to true, $y_{1}$ is removed by (4) and the six other variables in the first two clauses are set to false by (6). The last two clauses reduce to $\left(x, y_{2}, z_{3}\right)$ and $\left(x, y_{2}, z_{4}\right)$, but then $z_{3}=z_{4}$ by $(13)$ and (4). So we get a branching vector of at least $(8,2)$. 


$$
\begin{array}{cc}
\left(x, y_{1}, y_{3}, y_{5}, z_{1}\right) & \left(x, y_{1}\right)=\text { true }: y_{3}=y_{4}=y_{5}=y_{6}=z_{1}=z_{2}=\text { false }, \\
\left(x, y_{1}, y_{4}, y_{6}, z_{2}\right) & y_{1}=\bar{x}, z_{3} \stackrel{13}{=} z_{4} \\
\left(x, y_{2}, y_{3}, y_{6}, z_{3}\right) & \\
\left(x, y_{2}, y_{4}, y_{5}, z_{4}\right) &
\end{array}
$$

Figure 3: A $(4,0)$-occurrence $x$ with only ten variables.

Lemma 5. If a reduced formula does not satisfy any of the previous cases and it contains two variables $x$ and $y$ that occur in a clause together and they both also occur in a clause without the other, setting $(x, y)$ to true removes both $x$ and $y$.

Proof. When the algorithm sets $(x, y)$ to true then $y=\bar{x}$ by (4) and this makes $x$ a $(1,1)$-, $(2,1)$ - or $(2,2)$-occurrence, which we remove by resolution $(10$ or $(11))$.

\subsubsection{Two 5-clauses having exactly two variables in common}

Suppose $F$ contains two 5-clauses having two variables in common as the first two clauses in Figure 4(a). If both $x_{1}$ and $x_{2}$ occur in a clause

$$
\begin{array}{ll}
\left(x_{1}, x_{2}, y_{1}, y_{2}, y_{3}\right) & \left(x_{1}, x_{2}, y_{1}, y_{2}, y_{3}\right) \\
\left(x_{1}, x_{2}, y_{4}, y_{5}, y_{6}\right) & \left(x_{1}, x_{2}, y_{4}, y_{5}, y_{6}\right) \\
\left(x_{1}, y_{1}, C^{\prime}\right) & \left(x_{1}, z_{1}, z_{2}, z_{3}\right)
\end{array}
$$

(a) The third clause contains $y_{1}$.

(b) The third clause contains no $y$ 's.

Figure 4: Two 5-clauses having two variables in common.

without the other, the algorithm branches on $\left(x_{1}, x_{2}\right)$. Setting it to true removes all eight variables in the two clauses by Lemma 5, so this yields a branching vector of at least $(8,2)$.

By (9), at least one of $x_{1}$ and $x_{2}$ must occur in another clause, so assume we have another clause with $x_{1}$. Then $x_{1}$ is a $(3,0)$-occurrence and occurs in no other clauses. The third clause with $x_{1}$ can have at most one variable in common with each of the first two clauses apart from $x_{1}$ and if there is such a variable, the third clause must be a 5-clause.

The third clause contains $\mathbf{y}_{1}$ as in Figure 4(a), Now, $C^{\prime}$ contains three variables, one of which can be $y_{4}, y_{5}$ or $y_{6}$. If neither $y_{2}$ nor $y_{3}$ 
are unique, the algorithm branches on $\left(y_{2}, y_{3}\right)$; setting it to true removes both $y_{2}$ and $y_{3}$ by Lemma 5 , and $x_{1}, x_{2}$ and $y_{1}$ and setting $\left(y_{2}, y_{3}\right)$ to false removes $y_{2}$ and $y_{3}$, so we have the clauses in Figure 5; then we apply the reductions shown in the figure and remove $x_{2}$ and $y_{1}$. In total, we get a

$$
\begin{array}{llll}
\left(x_{1}, x_{2}, y_{1}\right) & \left(x_{1}, x_{2}, y_{1}\right) & \\
\left(x_{1}, x_{2}, y_{4}, y_{5}, y_{6}\right) & \stackrel{2 \times[13}{\longrightarrow} & \left(\bar{y}_{1}, y_{4}, y_{5}, y_{6}\right) & \stackrel{2 \times[10}{\longrightarrow} \\
\left(x_{1}, y_{1}, C^{\prime}\right) & \left(\bar{x}_{2}, C^{\prime}\right) & \left(x_{1}, y_{4}, y_{5}, y_{6}, C^{\prime}\right)
\end{array}
$$

Figure 5: The clauses from Figure $4(\mathrm{a})$ when $y_{2}=y_{3}=$ false.

branching vector of at least $(5,4)$.

In the other case, one of $y_{2}$ and $y_{3}$ (say $y_{3}$ ) is unique, but not $y_{2}$ by (9). Now, $y_{1}$ occurs in no other clauses; otherwise, $x_{1}$ and $y_{1}$ are two variables occurring twice together and both in a clause without the other, which is the first case above. The algorithm branches on $y_{2}$. Setting it to true removes at least seven variables, since $y_{2}$ occurs in another clause. Setting $y_{2}$ to false leaves the three clauses in Figure 6, where $y_{3}$ is unique, and $x_{1}$, $x_{2}$ and $y_{1}$ only occur in these three clauses; then we replace those three

\begin{tabular}{lll|}
$\left(x_{1}, x_{2}, y_{1}, y_{3}\right)$ & & $\left(x_{2}, y_{4}, y_{5}, y_{6}\right)$ \\
$\left(x_{1}, x_{2}, y_{4}, y_{5}, y_{6}\right)$ & $\rightarrow$ & $\left(y_{1}, C^{\prime}\right)$ \\
$\left(x_{1}, y_{1}, C^{\prime}\right)$ & & \\
\hline
\end{tabular}

Figure 6: Equivalent clauses.

clauses by the two clauses on the right: any truth assignment satisfying the original formula with $x_{1}$ true will satisfy the new formula if $y_{1}$ and $x_{2}$ are both changed to true and a satisfying assignment with $x_{1}$ false will also satisfy the new formula. On the other hand, a satisfying truth assignment to the new formula with both $x_{2}$ and $y_{1}$ true is a satisfying assignment to the original formula if $x_{1}$ is set to true and $x_{2}, y_{1}$ and $y_{3}$ are set to false. All other assignments satisfying the new formula can be changed to satisfy the original one by setting $x_{1}$ to false and choosing $y_{3}$ such that $\left(x_{2}, y_{1}, y_{3}\right)$ is satisfied. By doing this replacement, both $x_{1}$ and $y_{3}$ are removed, so we get a branching vector of at least $(7,3)$.

The third clause with $\mathrm{x}_{1}$ contains none of the $\mathrm{y}$ 's. If the third clause is not a 4-clause, the algorithm branches on $x_{1}$. If the third clause is a 3 -clause, this yields a branching vector of at least $(10,2)$ and if the 
third clause is at least a 5-clause, this yields a branching vector of at least $(12,1)$.

The remaining case is depicted in Figure 4(b) none of the $z_{i}$ 's is unique by $(9)$, since the two other clauses with $x_{1}$ contains $x_{2}$, which is in no other clauses. If one of $z_{1}, z_{2}$ and $z_{3}$ occurs three times, say $z_{1}$, the algorithm branches on $x_{1}, z_{1}$. Setting $x_{1}$ to true removes eleven variables, setting $z_{1}$ to true removes at least eight and setting both to false removes all four variables in the third clause by Lemma 5. So we get a branching vector of at least $(11,8,4)$.

In the last case, $z_{1}, z_{2}$ and $z_{3}$ all occur exactly twice in $F$; then the algorithm branches on $\left(x_{1}, z_{1}\right)$. All four variables in the third clause are removed in both branches by Lemma 5. Let the other clause containing $z_{1}$ be $\left(z_{1}, C^{\prime}\right)$. If it is a 3 -clause, we remove an extra variable setting $z_{1}$ to false. If the clause contains one of the $y$ 's, we get a clause where this $y$ occurs twice, when we set $\left(x_{1}, z_{1}\right)$ to true and the variable is removed by (5). Both cases result in a branching vector of at least $(5,4)$. Otherwise, $\left(z_{1}, C^{\prime}\right)$ is at least a 4 -clause containing none of the $y$ 's, but in that case when we set $\left(x_{1}, z_{1}\right)$ to true and apply (10), we get the clauses $\left(C^{\prime}, x_{2}, y_{1}, y_{2}, y_{3}\right)$ and $\left(C^{\prime}, x_{2}, y_{4}, y_{5}, y_{6}\right)$. These two clauses contain at least seven variables each and have all but three of them in common. We then further branch on $\left(C^{\prime}, x_{2}\right)$ and get a branching vector of at least $(6,4)$ (see Figure 2). In total, we get a branching vector of at least $(10,8,4)((4,4)$ followed by $(6,4)$ in one branch).

\subsubsection{Variables occurring three times}

If $F$ contains a variable occurring three times and not in three 4-clauses or two 4-clauses and a 5-clause, the algorithm branches on it. This yields a branching vector of at least $(7,4),(8,3),(9,2)$ or $(12,1)$ depending on the number of 3 -clauses the variable is in.

A $(3,0)$-occurrence in three 4-clauses. Suppose $F$ contains a $(3,0)$ occurrence $x$, which is in three 4-clauses as in Figure 7(a). Not all the clauses can contain a unique variable or $F$ would have been reduced by (9), so assume that the first clause contains no unique variables. If $y_{1}$ occurs with at least four other variables, we branch on $x, y_{1}$. Setting $x$ to true removes ten variables, setting $y_{1}$ to true removes at least eight and setting both to false removes four by Lemma 5. In total, we get a branching vector of at least $(10,8,4)$. 


$$
\begin{array}{lll}
\left(x, y_{1}, y_{2}, y_{3}\right) & \left(x, y_{1}, y_{2}, y_{3}\right) & \left(y_{1}, z_{1}, z_{2}, z_{3}\right) \\
\left(x, y_{4}, y_{5}, y_{6}\right) & \left(x, y_{4}, y_{5}, y_{6}\right) & \left(y_{2}, z_{4}, z_{5}, z_{6}\right) \\
\left(x, y_{7}, y_{8}, y_{9}\right) & \left(x, y_{7}, y_{8}, y_{9}\right) & \left(y_{3}, z_{7}, z_{8}, z_{9}\right)
\end{array}
$$

(a) A (3,0)-occurrence

(b) The variables $y_{1}, y_{2}$ and $y_{3}$ in three 4-clauses. are all $(2,0)$-occurrences.

Figure 7: A $(3,0)$-occurrence $x$ in three 4-clauses.

We can assume now, that $y_{1}, y_{2}$ and $y_{3}$ are all $(2,0)$-occurrences and that their other clause is at most a 4-clause; otherwise, we have the previous case. If $y_{1}$ is in a 3 -clause, the algorithm branches on $\left(x, y_{1}\right)$; in both branches all variables in the clause with $x$ and $y_{1}$ are removed by Lemma 5. Setting $\left(x, y_{1}\right)$ to false also removes one of the other variables from the 3 -clause by (4). This yields a branching vector of at least $(5,4)$.

If none of the previous cases applies, the other clauses containing $y_{1}$, $y_{2}$ and $y_{3}$ must be the ones in Figure 7(b), where some of the $z$ 's can be one of $y_{4}$ up to $y_{9}$ and some of them can be the same variable. By (9), at most one $z_{i}$ from each clause is unique. Suppose two $z_{i}$ 's from different clauses (say $z_{1}$ and $z_{4}$ ) are unique; then branching on $\left(y_{1}, y_{2}\right)$ will remove $x, y_{1}, y_{2}$ and $y_{3}$ in both branches and setting $\left(y_{1}, y_{2}\right)$ to true also makes $z_{1}$ and $z_{4}$ end up in the same clause and one is removed by (9). This also yields a branching vector of at least $(5,4)$.

We can assume now, that say $z_{1}, z_{2}$ and $z_{3}$ are not unique and if any of them are a $y$, then $z_{1}$ is $y_{4}$. The algorithm branches on $\left(y_{1}, z_{1}\right)$; in both branches $y_{1}, z_{1}, z_{2}$ and $z_{3}$ are removed. If $z_{1}$ was $y_{4}$, setting $\left(y_{1}, z_{1}\right)$ to true makes $y_{4}=\bar{y}_{1}$, so $x$ is false by (7). This yields a branching vector of at least $(5,4)$. If $z_{1}$ is not $y_{4}$, setting $\left(y_{1}, z_{1}\right)$ to false reduces the first clause with $x$ to $\left(x, y_{2}, y_{3}\right)$. The algorithm then branches on $x$, which yields a branching vector of at least $(9,3)$, since when $x$ is set to false, $y_{2}$ and $y_{3}$ are removed by Lemma 5. In total, this yields a branching vector of at least $(13,7,4)$.

A (3,0)-occurrence in two 4-clauses and a 5-clause. We have now removed all $(3,0)$-occurrences except those in two 4-clauses and one 5 -clause. If we have such a variable $x$ and one of the 4-clauses contains another $(3,0)$-occurrence $y_{1}$ we branch on $x, y_{1}$. Setting one of the $(3,0)$ occurrences to true removes eleven variables and setting both to false removes three, so we get a branching vector of at least $(11,11,3)$.

If we have a $(3,0)$-occurrence $x$ in two 4 -clauses and a 5 -clause and 
one of the 4-clauses contains only $(2,0)$-occurrences other than $x$, we branch as in the previous section. The only difference having a 5-clause instead of a 4-clause makes, is that setting $x$ to true removes an extra variable.

Sparse formulas. The only remaining case with variables occurring more than twice is a $(3,0)$-occurrence in two 4-clauses and one 5-clause, where both 4-clauses contain a unique variable and the other variables in the 4-clauses occur twice in $F$. Then $F$ is 5 -sparse, i.e., it contains at least four variables occurring at most twice for each variable occurring three times: we only count the variables in the 4-clauses. Each variable occurring three times occurs with two unique variables and four $(2,0)$ occurrences in the two 4-clauses. The $(2,0)$-occurrences might be in another clause with a variable occurring three times, but if this clause is a 4-clause it can contain at most one $(3,0)$-occurrence. Thus, we have at least two $(2,0)$-occurrences and two unique variables for each variable occurring at least three times, so the formula is 5-sparse and the algorithm solves the remaining formula in time $O\left(2^{n / 5}\right)$, where $n$ is the number of remaining variables.

\subsection{The algorithm for X3SAT}

In this section, we give our algorithm for X3SAT and show that it achieves a branching vector of $(12,4)$ corresponding to a running time of $O\left(2^{0.1379 n}\right)$. The previously best algorithms are by Kulikov [2] and Porschen, Randerath and Speckenmeyer [6] and have branching vector $(9,4)$ corresponding to a running time of $O\left(2^{0.1626 n}\right)$.

Extra reductions For X3SAT we have some extra reductions which are only needed to remove certain cycles. We do not use them in the reduction procedure, but rather apply them, when they are needed.

We are concerned with cycles because, if we have $k$ clauses and a variable in each is set to false, we would normally remove another variable from each of the remaining 2-clauses by (4), but if some of the clauses form a cycle on the variables not set to false, we may remove one less variable. As an example, $F\left[z_{1} \leftarrow z_{2}, z_{2} \leftarrow z_{3}, \ldots, z_{k-1} \leftarrow z_{k}, z_{k} \leftarrow z_{1}\right]$ only removes $k-1$ variables from $F$.

Reductions 19 and $(23)$ do not remove any variables and we will also refer to them as transformations. They are only used, when they allow 
us to apply another reduction or get a previous case: this means that we call the algorithm recursively on the reduced formula; either a variable is removed by the reduction procedure or the algorithm will branch on one of the previous cases.

The first two reductions remove $k$-cycles with $k$ or $k-1$ negations, the third some special $k$-cycles.

$$
\begin{aligned}
& \left(y_{1}, \bar{z}_{1}, z_{2}\right) \wedge\left(y_{2}, \bar{z}_{2}, z_{3}\right) \wedge \cdots \wedge\left(y_{k}, \bar{z}_{k}, z_{1}\right) \wedge F^{\prime} \rightarrow \\
& \qquad\left[y_{1}, y_{2}, \ldots, y_{k} \leftarrow \text { false }\right] \\
& \left(y_{1}, z_{1}, z_{2}\right) \wedge\left(y_{2}, \bar{z}_{2}, z_{3}\right) \wedge \cdots \wedge\left(y_{k}, \bar{z}_{k}, z_{1}\right) \wedge F^{\prime} \rightarrow F\left[z_{1} \leftarrow \text { false }\right] \\
& \left(\tilde{y}_{1}, \tilde{z}_{1}, z_{2}\right) \wedge\left(\tilde{y}_{2}, \tilde{z}_{2}, z_{3}\right) \wedge \cdots \wedge\left(\tilde{y}_{k}, \tilde{z}_{k}, z_{1}\right) \wedge F^{\prime} \rightarrow F[x \leftarrow \text { false }] \\
& \quad y_{i} \text { occur unnegated in a clause with the literal } x \text { and the } \\
& \text { parities of } k \text { and the number of negations are different }
\end{aligned}
$$

If there is a 3-cycle with one negation, we can use $(19)$ to either add a clause with the three variables not on the cycle or if all the four clauses are there remove any one of them. If the 3-cycle has a unique variable in the clause without the negated variable, we can remove this clause by (20). If $u$ is not unique, but also occurs in $\left(\bar{u}, y_{2}, y_{3}\right)$, but in no other clauses, we can remove that clause by (19) and still use this reduction.

$$
\begin{aligned}
\left(y_{1}, z_{1}, z_{2}\right) \wedge\left(y_{2}, z_{2}, z_{3}\right) & \wedge\left(y_{3}, \bar{z}_{3}, z_{1}\right) \wedge F^{\prime} \leftrightarrow \\
\left(y_{1}, z_{1}, z_{2}\right) & \wedge\left(y_{2}, z_{2}, z_{3}\right) \wedge\left(y_{3}, \bar{z}_{3}, z_{1}\right) \wedge\left(\bar{y}_{1}, y_{2}, y_{3}\right) \wedge F^{\prime} \\
\left(u, z_{1}, z_{2}\right) \wedge\left(y_{2}, z_{2}, z_{3}\right) & \wedge\left(y_{3}, \bar{z}_{3}, z_{1}\right) \wedge F^{\prime} \rightarrow \\
u \text { unique } & \left(y_{2}, z_{2}, z_{3}\right) \wedge\left(y_{3}, \bar{z}_{3}, z_{1}\right) \wedge F^{\prime}
\end{aligned}
$$

If two 3-cycles without negations share two clauses, we can reduce the formula by 21 or 22 .

$$
\begin{gathered}
\left(y_{1}, z_{1}, z_{2}\right) \wedge\left(y_{2}, z_{2}, z_{3}\right) \wedge\left(y_{3}, z_{3}, z_{1}\right) \wedge\left(y_{1}, y_{2}, z_{4}\right) \wedge F^{\prime} \rightarrow \\
\left(z_{1}, z_{2}, z_{3}\right) \wedge F^{\prime}\left[y_{1} \leftarrow z_{3}, y_{2} \leftarrow z_{1}, y_{3} \leftarrow z_{2}, z_{4} \leftarrow z_{2}\right] \\
\left(y, z_{1}, z_{2}\right) \wedge\left(y, z_{3}, z_{4}\right) \wedge\left(y, z_{5}, z_{6}\right) \wedge\left(z_{1}, z_{3}, z_{5}\right) \wedge F^{\prime} \rightarrow F[y \leftarrow \text { false }]
\end{gathered}
$$

If we have a 3 -cycle with no negations and one of the variables is unique, we can transform the formula by 23 .

$$
\begin{aligned}
&\left(y_{1}, z_{1}, z_{2}\right) \wedge\left(y_{2}, z_{2}, z_{3}\right) \wedge\left(u, z_{3}, z_{1}\right) \wedge F^{\prime} \rightarrow \\
& u \text { unique } \\
&\left(y_{1}, z_{1}, z_{2}\right) \wedge\left(y_{2}, z_{2}, z_{3}\right) \wedge\left(\bar{y}_{1}, z_{3}, u\right) \wedge F^{\prime}
\end{aligned}
$$


If there is a 4-cycle with two negations and the negated variables occur nowhere else, the formula can be reduced by (24) or 25) ( $w$ is a new variable).

$$
\begin{aligned}
\left(y_{1}, z_{1}, z_{2}\right) \wedge\left(y_{2}, \bar{z}_{2}, z_{3}\right) \wedge( & \left(y_{3}, z_{3}, z_{4}\right) \wedge\left(y_{4}, \bar{z}_{4}, z_{1}\right) \\
z_{2}, z_{4} \notin V\left(F^{\prime}\right) & \wedge F^{\prime} \rightarrow \\
\left(w, z_{1}, z_{3}\right) & \wedge\left(\bar{w}, y_{1}, y_{2}\right) \wedge\left(\bar{w}, y_{3}, y_{4}\right) \wedge F^{\prime} \\
\left(y_{1}, z_{1}, z_{2}\right) \wedge\left(y_{2}, z_{2}, z_{3}\right) \wedge\left(y_{3}, \bar{z}_{3}, z_{4}\right) & \wedge\left(y_{4}, \bar{z}_{4}, z_{1}\right) \wedge F^{\prime} \rightarrow \\
z_{3}, z_{4} \notin V\left(F^{\prime}\right) & \\
\left(y_{1}, z_{1}, z_{2}\right) & \wedge\left(\bar{y}_{1}, y_{2}, w\right) \wedge\left(\bar{w}, y_{3}, y_{4}\right) \wedge F^{\prime}
\end{aligned}
$$

Lemma 6. Reductions (16) to (25) are sound.

Proof. In (16), the $z$ 's are either all true or all false or there would be a clause with two true literals. So the $y$ 's must be false.

In (17), if $z_{1}$ is true $z_{2}$ must be false, but then also $z_{3}$ must be false and the remaining $z$ 's must be false; then in the last clause, both $z_{1}$ and $\bar{z}_{k}$ are true, which is a contradiction.

Reduction (18) is proved with a simple counting argument: let $n_{1}$ be the number of $y$ 's that are negated and $n_{2}$ the number of $z$ 's occurring negated. In a satisfying assignment with $x$ true, $n_{1}$ of the clauses will be satisfied by the $y$ 's, $n_{2}$ of the clauses will be satisfied by the $z$ 's occurring negated, and an even number of clauses will be satisfied by the $z$ 's occurring only unnegated. This is only possible, if the parities of $k$ and $n_{1}+n_{2}$ are the same.

To prove the soundness of (19), we prove that all assignments satisfying the left hand side of the reduction will also satisfy the right hand side (the opposite is trivial). If $y_{1}$ is true, $z_{1}$ and $z_{2}$ must be false and $y_{2}$ and $y_{3}$ must have different values, so $\left(\bar{y}_{1}, y_{2}, y_{3}\right)$ is satisfied. If $y_{1}$ is false, $z_{2}=\bar{z}_{1}$ and $y_{2}$ and $y_{3}$ are both false by (8), so $\left(\bar{y}_{1}, y_{2}, y_{3}\right)$ is also satisfied in this case.

As $u$ is unique in (20), the first clause just ensures that $z_{1}$ and $z_{2}$ are not both true, but this is also ensured by the two other clauses, as $z_{1}$ is in a clause with $\bar{z}_{3}$ and $z_{2}$ with $z_{3}$, so we can remove the first clause.

In (21), setting $y_{1}=\bar{z}_{3}$ leads to a contradiction: by the first and second clause $z_{2}$ is false and by the second and fourth clause $y_{2}$ is false, which makes $z_{3}$ true; now, $z_{1}$ should both be false (by the third clause) and true (by the first clause), so in a satisfying assignment $y_{1}=z_{3}$; then $y_{2}=z_{1}$ by the first and second clause, $z_{4}=z_{2}$ by the second and fourth 
clause and $y_{3}=z_{2}$. With these substitutions all four clauses have become $\left(z_{1}, z_{2}, z_{3}\right)$, and three of the copies are discarded.

In (22), setting $y$ to true will set all the $z$ 's to false, but then the clause with only $z$ 's is not satisfied; thus, $y$ must be false.

In (23), the last clause on the left just ensures that not both $z_{1}$ and $z_{3}$ are true and the last clause on the right that not both $\bar{y}_{1}$ and $z_{3}$ are true. But by the first two clauses $z_{1}$ and $z_{3}$ are both true if and only if $\bar{y}_{1}$ and $z_{3}$ are both true, so we can replace the last clause on the left by the last clause on the right.

In (24), as $z_{2}$ does not occur elsewhere the first two clauses just ensure, that exactly one of $y_{1}, y_{2}, z_{1}$ and $z_{3}$ is true. This is also achieved by the clauses $\left(w_{1}, z_{1}, z_{3}\right)$ and $\left(\bar{w}_{1}, y_{1}, y_{2}\right)\left(w_{1}\right.$ is a new variable). Similarly, the last two clauses can be replaced by $\left(w_{2}, z_{1}, z_{3}\right)$ and $\left(\bar{w}_{2}, y_{3}, y_{4}\right)$, but then $w_{1}=w_{2}$ by (14) and we get the three clauses on the right hand side of (24). Similarly in (25), the last three clauses just ensure that exactly one of $y_{2}, y_{3}, y_{4}, z_{1}$ and $z_{2}$ is true, but this can also be expressed by the clauses $\left(w_{1}, z_{1}, z_{2}\right),\left(\bar{w}_{1}, y_{2}, w_{2}\right)$ and $\left(\bar{w}_{2}, y_{3}, y_{4}\right)$; then $w_{1}=y_{1}$ by (14), so we get the three clauses on the right hand side.

\subsubsection{General branching}

Now, we state our algorithm for X3SAT. If we have an $(a, b)$-occurrence $x$ occurring in the clauses in Figure 8, we let $Y_{1}=\left\{y_{1}, y_{2}, \ldots\right\}$ be the set of variables that occur in a clause with $x, Y_{2}=\left\{y_{1}^{\prime}, y_{2}^{\prime}, \ldots\right\}$ those that occur in a clause with $\bar{x}$ and $Y=Y_{1} \cup Y_{2}$. We let $y$ 's be variables in $Y_{1}, y^{\prime}$ 's be

\begin{tabular}{|llrl}
\hline$\left(x, y_{1}, y_{2}\right)$ & $\left(\bar{x}, y_{1}^{\prime}, y_{2}^{\prime}\right)$ & $x=$ true $: y_{1}=y_{2}=\cdots=y_{2 a-1}=y_{2 a}=$ false \\
$\left(x, y_{3}, y_{4}\right)$ & $\left(\bar{x}, y_{3}^{\prime}, y_{4}^{\prime}\right)$ & $y_{2}^{\prime}=\bar{y}_{1}^{\prime}, y_{4}^{\prime}=\bar{y}_{3}^{\prime}, \ldots, y_{2 b}=\bar{y}_{2 b-1}^{\prime}$ \\
$\vdots$ & $\vdots$ & $x=$ false $:$ & $y_{2}=\bar{y}_{1}, y_{4}=\bar{y}_{3}, \ldots, y_{2 b}=\bar{y}_{2 b-1}$ \\
$\left(x, y_{2 a-1}, y_{2 a}\right)$ & $\left(\bar{x}, y_{2 b-1}^{\prime}, y_{2 b}^{\prime}\right)$ & $y_{1}^{\prime}=y_{2}^{\prime}=\cdots=y_{2 a-1}^{\prime}=y_{2 a}^{\prime}=$ false
\end{tabular}

Figure 8: Branching on an $(a, b)$-occurrence $x$.

variables in $Y_{2}, z$ 's be variables that are not $x$ and not in $Y$ and $w$ 's be variables that are not $x$. By looking at $\bar{x}$ instead of $x$ we swap $Y_{1}$ and $Y_{2}$.

If we branch on $x$ in Figure 8, we get a branching vector of at least $(2 a+b+1,2 b+a+1)$ from the above clauses. If $a+b \geq 5$, this yields a branching vector of at least $(11,6),(10,7)$ or $(9,8)$. For variables occurring fewer times, we also need to consider the other clauses in which 
the $y$ 's occur. We start with a lemma showing some cases, in which we can reduce $F$.

Lemma 7. If a reduced formula $F$ contains a clause with three variables from $Y$ that is not $\left(\bar{y}_{1}, y_{3}, y_{5}\right)$ or if $F$ contains a clause $\left(\bar{y}_{1}, \bar{y}_{3}, z_{1}\right)$ or $\left(\tilde{y}_{1}, \tilde{y}_{1}^{\prime}, z_{1}\right)$, where at least one of $y_{1}$ and $y_{1}^{\prime}$ is negated, $F$ can be reduced.

Proof. If $F$ contains a clause with $y_{1}$ and $y_{1}^{\prime}$ where at least one of them is negated or with $y_{1}$ and $y_{3}$ where both of them are negated, it contains a 3-cycle with two or three negations and we reduce it by $(16)$ or $(17)$. If $F$ contains the clause $\left(y_{1}, y_{1}^{\prime}, y_{3}\right)$, we add the clause $\left(y_{2}, y_{2}^{\prime}, \bar{y}_{3}\right)$ by $(19)$ and have the previous case. The only case left is if $F$ contains the clause $\left(y_{1}, y_{3}, y_{5}\right)$; then $x$ must be false by 22 .

If $x$ is a $(3,1)$-occurrence or a $(2,2)$-occurrence, one of the variables in one clause with $\bar{x}$ say $y_{1}^{\prime}$ must occur in another clause by (15) and the clause must be $\left(\tilde{y}_{1}^{\prime}, w, z_{1}\right)$ by Lemma 7, since $y_{1}^{\prime}$ can not occur with two other $y^{\prime \prime}$ s as $x$ only occurs negated in at most two clauses. From the clauses in Figure 8, we get a branching vector of at least $(8,6)$ or $(7,7)$, but setting $x$ to false, also removes $z_{1}$ by (1) or (4) and we get a branching vector of at least $(8,7)$. Now, we have removed all variables occurring at least four times in the formula, except $(4,0)$-occurrences.

\subsubsection{Branching on $(2,1)$-occurrences}

By (15), at least one $y$ from each clause with $x$ and two from one are in other clauses. We want to show, that in all cases we can either reduce $F$ or branch on $x$ and get a branching vector of at least $(8,7)$ or $(9,6)$. From the clauses with $x$, we get a branching vector of at least $(6,5)$ (see Figure 8). We want to show, that we can always remove at least four more variables in total in the two branches from the other clauses with the variables from $Y$. First, we prove two lemmas showing, when we can reduce the formula.

Lemma 8. If a reduced formula $F$ contains the clause $\left(\tilde{y}_{1}, \tilde{y}_{1}^{\prime}, z_{1}\right)$ and a clause containing $z_{1}$ and a variable from $\left\{y_{1}, y_{2}, y_{1}^{\prime}, y_{2}^{\prime}\right\}$ and the clauses are not $\left(y_{1}, y_{1}^{\prime}, z_{1}\right)$ and $\left(y_{2}, y_{2}^{\prime}, \bar{z}_{1}\right), F$ can be reduced.

Proof. By Lemma 7, if the first clause is not $\left(y_{1}, y_{1}^{\prime}, z_{1}\right), F$ can be reduced. If $F$ does not contain the clause $\left(y_{2}, y_{2}^{\prime}, \bar{z}_{1}\right)$, we add it by 19 and since the second clause was not this one we have two clauses sharing at least two variables and we reduce $F$ by one of (7), (8) or (14). 


$$
\begin{array}{lll}
\left(x, y_{1}, y_{2}\right)\left(y_{1}, y_{1}^{\prime}, z_{1}\right) & \left(x, y_{1}, y_{2}\right)\left(\bar{y}_{1}, y_{3}, z_{1}\right) & \left(x, y_{1}, y_{2}\right)\left(y_{1}, y_{3}, z_{1}\right) \\
\left(x, y_{3}, y_{4}\right) & \left(x, y_{3}, y_{4}\right) & \left(x, y_{3}, y_{4}\right) \\
\left(\bar{x}, y_{1}^{\prime}, y_{2}^{\prime}\right) & \left(\bar{x}, y_{1}^{\prime}, y_{2}^{\prime}\right) & \left(\bar{x}, y_{1}^{\prime}, y_{2}^{\prime}\right)
\end{array}
$$

(a) (c)

Figure 9: A $(2,1)$-occurrence $x$ and a clause with two variables from $Y$.

Lemma 9. If a reduced formula $F$ contains the clause $\left(\tilde{y}_{1}, \tilde{y}_{3}, z_{1}\right)$ and a clause containing $z_{1}$ and a variable from $\left\{y_{1}, y_{2}, y_{3}, y_{4}\right\}$ and the clauses are not $\left(\bar{y}_{1}, y_{3}, z_{1}\right)$ and $\left(y_{2}, \bar{y}_{4}, z_{1}\right), F$ can be reduced.

Proof. By Lemma 7, at most one of $y_{1}$ and $y_{3}$ is negated. Suppose one is negated, then we have the clauses in Figure 9(b). If the other clause is not $\left(y_{2}, \bar{y}_{4}, z_{1}\right)$, we add this clause by $(19)$. Now, the other clause with $z_{1}$ and one of the $y$ 's will share at least two variables with one of the other two clauses with $z_{1}$ and we reduce by one of (7), (8) or (14).

Suppose we have the clauses in Figure 9(c). By symmetry, we can assume that the second clause with $z_{1}$ is $\left(\tilde{y}_{2}, \tilde{z}_{1}, w\right)$. If none of $y_{2}$ and $z_{1}$ is negated, we reduce $F$ by (21) and if both are negated there is a 3-cycle with two negations, so we reduce $F$ by (17). If only one is negated we have a 3-cycle with one negation and we add a clause with $x$ and $y_{3}$ by (19), where one of them is negated and reduce $F$ by (7).

Note, that we can not have a clause with three variables from $Y$ when $x$ is a $(2,1)$-occurrence, by Lemma 7 .

Suppose $F$ contains the clauses in Figure 9(a), using (19) we can transform $F$ to contain one or both of $\left(y_{1}, y_{1}^{\prime}, z_{1}\right)$ and $\left(y_{2}, y_{2}^{\prime}, \bar{z}_{1}\right)$. Now, if there is only one other clause, $C$, with variables from $Y$, we reduce $F$ : we can choose to let $F$ contain one of the above clauses such that at most three variables from $Y$ occur in this clause and $C$ and reduce $F$ by (15). Suppose that $F$ contains two other clauses with variables from $Y$ and that they contain different $z$ 's, none of which is $z_{1}$; then branching on $x$ yields $(8,7)$ or $(9,6)$ : in both branches, $y_{1}$ or $y_{1}^{\prime}$ is set to false in $\left(y_{1}, y_{1}^{\prime}, z_{1}\right)$, so $z_{1}$ is removed by (4). In each of the other clauses, one variable is removed when the $y$ is set to either true or false.

Suppose, on the other hand, that $F$ does not contain two such clauses; then $z_{1}$ is in no more clauses with $y_{1}, y_{2}, y_{1}^{\prime}$ or $y_{2}^{\prime}$ by Lemma 8 and if it is in a clause with $y_{3}$, the clause contains no other variable from $Y$. Suppose $F$ contains the clause $\left(\tilde{y}_{3}, \tilde{z}_{1}, z_{2}\right)$; then $z_{1}$ can be in no more clauses with variables from $Y$. F must contain another clause with a variable from $Y$ 
and since it does not contain $z_{1}$ or any $z_{3}$, it must contain $z_{2}$ and two variables from $Y$. Using Lemma 8 and 9 , we have that this clause must be $\left(y_{1}, y_{2}^{\prime}, z_{2}\right)$ or $\left(y_{2}, y_{1}^{\prime}, \bar{z}_{2}\right)$ (or $\left(y_{1}^{\prime}, y_{2}, z_{2}\right)$ or $\left(y_{2}^{\prime}, y_{1}, \bar{z}_{2}\right)$, which we handle similarly) and we add the other by (19). Now, we have the clauses in Figure 10, but then we have a 3-cycle with two negations which we remove

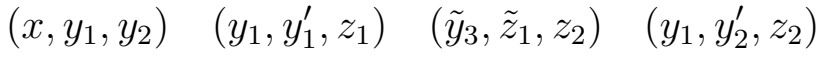

$$
\begin{aligned}
& \left(x, y_{3}, y_{4}\right) \quad\left(y_{2}, y_{2}^{\prime}, \bar{z}_{1}\right) \quad\left(y_{2}, y_{1}^{\prime}, \bar{z}_{2}\right) \\
& \left(\bar{x}, y_{1}^{\prime}, y_{2}^{\prime}\right)
\end{aligned}
$$

Figure 10: A special case for $(2,1)$-occurrence $x$, in which we can reduce.

by (17): the 3-cycle contains the bottom clause in the fourth column, the clause in the third column and one of the clauses in the second column (which one depends on whether $z_{1}$ is negated in $\left(\tilde{y}_{3}, \tilde{z}_{1}, z_{2}\right)$ ).

If the two other clauses with variables from $Y$ do not contain $z_{1}$ and not two different $z$ 's, they must be of the form $\left(\bar{y}_{1}, y_{3}, z_{2}\right)$ and $\left(y_{2}, \bar{y}_{4}, z_{2}\right)$ or $\left(y_{3}, y_{1}^{\prime}, z_{2}\right)$ and $\left(y_{4}, y_{2}^{\prime}, \bar{z}_{2}\right)$ by Lemma 8 and 9 , but then we remove one of the clauses by (19) and have the previous case with only one other clause with variables from $Y$. This completes all cases with the clauses in Figure 9(a).

Suppose $F$ contains the clauses in Figure $9(\mathrm{~b})$. If $y_{1}$ is only in the clause $\left(\bar{y}_{1}, y_{3}, z_{1}\right)$ and the one with $x$, we replace the first clause by $\left(y_{2}, \bar{y}_{4}, z_{1}\right)$ by applying (19) twice and reduce $F$ by $(20)$. If $y_{1}$ is in another clause, it must be a $(2,1)$-occurrence and since $x$ and $y_{3}$ are in a clause together, we have the previous case.

Suppose $F$ contains the clauses in Figure 9(c). If $y_{2}$ is unique, we transform $F$ by (23) and replace the clause $\left(x, y_{1}, y_{2}\right)$ by $\left(x, \bar{z}_{1}, y_{2}\right)$, but then we have the previous case. Now, $y_{2}$ and symmetrically $y_{4}$ must occur in another clause. If they occur together in a clause, they must occur unnegated or we have the previous case, but then we reduce $F$ by (21). The clauses with $y_{2}$ and $y_{4}$ do not contain $z_{1}$ by Lemma 9 and no $y^{\prime}$ or negated $y$ by the previous cases, so they must contain two different $z$ 's. As before, this yields a branching vector of at least $(9,6)$ branching on $x$.

If no two variables from $Y$ occur in the same clause, we get $(9,6)$ or $(8,7)$ branching on $x$ : at least two of the variables in $Y_{1}$ and at least one of those in $Y_{2}$ must be in another clause, which removes at least two extra variables in the true branch and one in the false branch. Now, at least one more of the variables from $Y$ must be in another clause. This removes an extra variable in one branch, unless there are three clauses 
with a variable from $Y_{1}$ and the $z$ 's in these three clauses form a 3-cycle, but then $F$ can be reduced by Lemma 10 .

Lemma 10. If a reduced formula $F$ contains a 3-cycle consisting of the clauses $\left(\tilde{y}_{1}, \tilde{z}_{1}, z_{2}\right),\left(\tilde{y}_{2}, \tilde{z}_{2}, z_{3}\right)$ and $\left(\tilde{y}_{3}, \tilde{z}_{3}, z_{1}\right)$, F can be reduced.

Proof. If more than one of the $z$ 's are negated, $F$ is reduced by 16 or (17), and if exactly one is negated, we use (19) to add a clause with $y_{1}, y_{2}$ and $y_{3}$, but $y_{1}$ and $y_{2}$ are already together in a clause with $x$, so we can reduce $F$. If none of the $z$ 's is negated we look at whether $y_{1}$ and $y_{2}$ are negated. If both are negated we have a 3-cycle with two negations, which we reduce by (17) and if none of them is negated, the formula is reduced by (21). If either $y_{1}$ or $y_{2}$ is negated, we add a clause with $x, z_{1}$ and $z_{3}$ by $(19)$ where one of $z_{1}$ and $z_{3}$ are negated and reduce the formula by (7).

\subsubsection{Branching on (4,0)-occurrences}

To get the desired branching vector for $(4,0)$-occurrences, we show that if there are no variables occurring both unnegated and negated except $(1,1)$-occurrences, we can extend Lemma 7 and reduce in all cases with a clause with three $y$ 's.

Lemma 11. If a reduced formula $F$ containing only $(a, 0)$ - and $(1,1)$ occurrences contains a clause $\left(\tilde{y}_{1}, \tilde{y}_{3}, \tilde{y}_{5}\right)$ or a clause $\left(\bar{y}_{1}, \tilde{y}_{3}, w\right), F$ can be reduced.

Proof. The only case not covered by Lemma 7 is if $F$ contains the clause $\left(\bar{y}_{1}, y_{3}, w\right)$, where $w$ is either $y_{5}$ or a $z$. Now, $y_{1}$ must be a $(1,1)$ occurrences so we replace this clause by $\left(y_{2}, \bar{y}_{4}, w\right)$ by applying $(19)$ twice and then reduce $F$ by 20 .

If $x$ is a $(4,0)$-occurrence, branching on $x$ yields a branching vector of at least $(9,5)$ from the clauses in Figure 8 . At least five of the $y$ 's must occur in another clause, but then they must occur with at least two different $z$ 's or we reduce $F$ by Lemma 9 or 11 . When we set $x$ to true all the $y$ 's are false and at least two $z$ 's are removed and we get a branching vector of at least $(11,5)$.

\subsubsection{Branching on (3,0)-occurrences}

When we look at a $(3,0)$-occurrence $x$, we know by Lemma 11 that no three variables from $Y$ occur together and if two occur together they must both occur unnegated. 
F contains a clause with two variables from Y. Suppose $F$ contains the clause $\left(y_{1}, y_{3}, z_{1}\right)$ and two of $y_{2}, y_{4}$ and $z_{1}$ are unique, then we use (23) (with the other unique variable as $y_{2}$ in (23)) and remove one of the unique variables by (20), and if only one of them is negated, we use (23) and get a $(2,1)$-occurrence, which is a previous case. If $z_{1}$ is in a clause with any of the variables $y_{1}, y_{2}, y_{3}$ or $y_{4}$, we reduce $F$ by Lemma 9 . If two of $y_{1}, y_{2}, y_{3}$ and $y_{4}$ occur together in a second clause they must both be unnegated by Lemma 11, but then we reduce $F$ by (14), (21) or (22), so we must have the clauses in Figure 11, where $z_{2}$ is a new

$$
\begin{array}{ll}
\left(x, y_{1}, y_{2}\right)\left(y_{1}, y_{3}, z_{1}\right) & y_{2}=\text { true }: x=y_{1}=\text { false, } y_{4}=\bar{y}_{3}, y_{6}=\bar{y}_{5}, z_{1}=\bar{y}_{3} \\
\left(x, y_{3}, y_{4}\right)\left(\tilde{y}_{2}, w, z_{2}\right) & y_{2}=\text { false }: y_{1}=\bar{x}, y_{3} \stackrel{(7)}{=} \text { false, } y_{4}=\bar{x}, z_{1}=\bar{y}_{1} \\
\left(x, y_{5}, y_{6}\right) & \tilde{y}_{2}=\text { true }: w=z_{2}=\text { false } \\
\tilde{y}_{2}=\text { false }: z_{2}=\bar{w}
\end{array}
$$

Figure 11: A clause with two $y$ 's. We branch on $y_{2}$.

variable, and $w$ can be either $y_{5}$ (it can not be negated by Lemma 11 ) or $z_{3}$ (another new variable). If $w$ is $y_{5}$, the clause has two $y$ 's and $z_{2}$ can not be unique by the above. The algorithm branches on $y_{2}$. Let us first look at what happens in the first four clauses; setting $y_{2}$ to true removes the six variables depicted in Figure 11, and setting $y_{2}$ to false makes $y_{1}=\bar{x}$. Then we have the two clauses $\left(x, y_{3}, y_{4}\right)$ and $\left(\bar{x}, y_{3}, z_{1}\right)$ which makes $y_{3}$ false by (7) and we remove the last two variables in Figure 11 for at total of five variables. Now, we look at what happens with the clause $\left(\tilde{y}_{2}, w, z_{2}\right)$; when $\tilde{y}_{2}$ is set to false, we remove $z_{2}$, and when $\tilde{y}_{2}$ is set to true, both $w$ and $z_{2}$ are set to false. This removes two additional variables in this branch: $w$ is either $y_{5}$ or $z_{3}$, but neither has gotten a value in any of the branches. Now, either $z_{2}$ is in another clause or $w$ is $z_{3}$ and is in another clause. This clause can at most contain one of $y_{1}$, $y_{2}, y_{3}, y_{4}$ or $z_{1}$ by Lemma 9 , so we remove an extra variable from this clause when $z_{2}$ or $z_{3}$ is set to false. In total, we remove three variables in one branch and one in the other. This yields a branching vector of at least $(9,6)$ or $(8,7)$.

Cycles At this point, the only clauses containing $y$ 's except the clauses with $x$ contain only one $y$. By (15), at least four of the $y$ 's are not unique, so there must be at least four such clauses. We want to branch on $x$; when we set it to true, all the $y$ 's are set to false and the literals from 
clauses with unnegated $y$ 's are set to the negation of each other and the ones from clauses with negated $y$ 's are set to false. This removes at least as many extra variables as there are clauses with $y$ 's, unless some of these clauses form a cycle as in Figure 12. We are only concerned with 3-, 4-

$$
\begin{array}{rrr}
\left(x, y_{1}, y_{2}\right)\left(\tilde{y}_{1}, \tilde{z}_{1}, z_{2}\right) & \left(x, y_{1}, y_{2}\right)\left(\tilde{y}_{1}, \tilde{z}_{1}, z_{2}\right) & \left(x, y_{1}, y_{2}\right)\left(\tilde{y}_{1}, \tilde{z}_{1}, z_{2}\right) \\
\left(x, y_{3}, y_{4}\right)\left(\tilde{y}_{i}, \tilde{z}_{2}, z_{3}\right) & \left(x, y_{3}, y_{4}\right)\left(\tilde{y}_{i}, \tilde{z}_{2}, z_{3}\right) & \left(x, y_{3}, y_{4}\right)\left(\tilde{y}_{i}, \tilde{z}_{2}, z_{3}\right) \\
\left(x, y_{5}, y_{6}\right)\left(\tilde{y}_{j}, \tilde{z}_{3}, z_{1}\right) & \left(x, y_{5}, y_{6}\right)\left(\tilde{y}_{j}, \tilde{z}_{3}, z_{4}\right) & \left(x, y_{5}, y_{6}\right)\left(\tilde{y}_{j}, \tilde{z}_{3}, z_{4}\right) \\
& \left(\tilde{y}_{k}, \tilde{z}_{4}, z_{1}\right) & \left(\tilde{y}_{k}, \tilde{z}_{4}, z_{5}\right) \\
& & \left(\tilde{y}_{l}, \tilde{z}_{5}, z_{1}\right)
\end{array}
$$
(a) 3-cycle.
(b) 4-cycle.
(c) 5-cycle.

Figure 12: Cycles.

and 5-cycles; we can not have 2-cycles, as these would have been removed by (7), (8) or (14) and if we have at least a 6-cycle, we remove at least five $z$ 's, when we set $x$ to true, but this yields a branching vector of at least $(12,4)$, which is what we are after.

Lemma 12. If a reduced formula does not satisfy any of the previous cases, the same y can not occur twice in a 3-, 4- or 5-cycle.

Proof. If a $y$ occurs twice in a 3-, 4- or 5-cycle, it occurs at least three times in $F$ so it must be a $(3,0)$-occurrence. It can not occur in two neighbouring clauses on the cycle by (7) and (14); since we are dealing with at most 5 -cycles, it must then occur in two clauses $\left(y_{1}, \tilde{z}_{1}, z_{2}\right)$ and $\left(y_{1}, \tilde{z}_{3}, z_{4}\right)$, where $z_{2}$ and $z_{3}$ are together in another clause on the cycle. Then $y_{1}$ is a $(3,0)$-occurrence with two of the variables it occurs with occurring together in another clause, but that is a previous case.

Lemma 13. If a reduced formula $F$ containing only $(a, 0)$ - and $(1,1)$ occurrences contains two clauses $\left(\tilde{y}_{i}, z_{1}, z_{2}\right)$ and $\left(\tilde{y}_{j}, \tilde{z}_{2}, z_{3}\right)$, where at least two of $y_{i}, y_{j}$ and $z_{2}$ are negated, we can reduce $F$.

Proof. If $y_{i}$ and $y_{j}$ are from the same clause with $x$, that clause and the two clauses in the lemma form a 3-cycle with two or three negations, so we can reduce $F$ by $(16)$ or $(17)$. If $y_{i}$ and $y_{j}$ are from different clauses with $x$, the clauses form a 4-cycle with two or three negations together with the two clauses where $y_{i}$ and $y_{j}$ occur with $x$, and we reduce $F$ by (17), 24) or (25), since we do not have $(2,1)$-occurrences.

In the following, we show how to deal with the remaining cases of 3-, 4- and 5-cycles. 
3-cycles. If $F$ contains a 3-cycle as in Figure 12(a), the $y$ 's must be from different clauses with $x$ by Lemma 10 . If the cycle contains more than one negated $z$, the formula is reduced by (16) or (17) and if there is exactly one negated $z$, we add a clause with three $y$ 's by (19). If this clause does not contain exactly one negation, we reduce $F$ by Lemma 7 ; otherwise, the negated $y$ has become a $(2,1)$-occurrence, which is a previous case. Now, suppose that none of the $z$ 's is negated; if at least two of the $y$ 's are negated, we reduce $F$ by Lemma 13 and if none of the $y$ 's is negated we reduce $F$ by (18). So assume the cycle consists of the clauses in Figure 13 , then we branch on $y_{1}$ and get a branching vector of at least

$$
\begin{aligned}
& \left(x, y_{1}, y_{2}\right)\left(\bar{y}_{1}, z_{1}, z_{2}\right) y_{1}=\text { true }: x=y_{2}=\text { false, } y_{4}=\bar{y}_{3}, y_{6}=\bar{y}_{5}, z_{2}=\bar{z}_{1} \\
& \left(x, y_{3}, y_{4}\right)\left(y_{3}, z_{2}, z_{3}\right) \quad z_{3} \stackrel{\text { [7 }}{=} \text { false, } y_{3}=\bar{z}_{2}, y_{5}=\bar{z}_{1} \\
& \left(x, y_{5}, y_{6}\right)\left(y_{5}, z_{3}, z_{1}\right) y_{1}=\text { false }: y_{2}=\bar{x}, z_{1}=z_{2}=\text { false } \\
& y_{3}=y_{5}=\bar{z}_{3}, y_{4}=y_{6}
\end{aligned}
$$

Figure 13: A 3-cycle with only $y_{1}$ negated. We branch on $y_{1}$.

$(9,7)$, as shown in the figure. The (7) above the equality means, that this follows from (7): since $z_{2}=\bar{z}_{1}$, we get two clauses with $z_{1}$ and $z_{3}$ and $z_{1}$ is negated in one of them.

4-cycles. Suppose $F$ contains a 4-cycle as in Figure 12(b). If there are two or more negated $z$ 's, we reduce the formula by $(16),(17),(24)$ or $(25)$, since $F$ contains no $(2,1)$-occurrences. If there is only one negated $z$, the two $y$ 's in the clauses with the negated variable must be unnegated by Lemma 13 , let these clauses be $\left(y_{1}, z_{1}, z_{2}\right)$ and $\left(y_{i}, \bar{z}_{2}, z_{3}\right)$. If an even number of the $y$ 's are negated, we reduce the formula by (18); otherwise, there must be exactly one negated $y$ and the cycle looks like the one in Figure 14(a), but then $x=z_{1}$. Suppose $x=\bar{z}_{1}$; then we replace $z_{1}$ by $\bar{x}$

$$
\begin{array}{rrr}
\left(x, y_{1}, y_{2}\right)\left(y_{1}, z_{1}, z_{2}\right) & \left(x, y_{1}, y_{2}\right)\left(y_{1}, z_{1}, z_{2}\right) & \left(x, y_{1}, y_{2}\right)\left(y_{1}, z_{1}, z_{2}\right) \\
\left(x, y_{3}, y_{4}\right)\left(y_{i}, \bar{z}_{2}, z_{3}\right) & \left(x, y_{3}, y_{4}\right)\left(y_{2}, z_{2}, z_{3}\right) & \left(x, y_{3}, y_{4}\right)\left(y_{3}, z_{2}, z_{3}\right) \\
\left(x, y_{5}, y_{6}\right)\left(\bar{y}_{j}, z_{3}, z_{4}\right) & \left(x, y_{5}, y_{6}\right)\left(y_{3}, z_{3}, z_{4}\right) & \left(x, y_{5}, y_{6}\right)\left(y_{2}, z_{3}, z_{4}\right) \\
\left(y_{k}, z_{4}, z_{1}\right) & \left(y_{i}, z_{4}, z_{1}\right) & \left(y_{4}, z_{4}, z_{1}\right)
\end{array}
$$
(a) $x=z_{1}$.
(b) $z_{2}=z_{4}$.
(c) Remove last clause.

Figure 14: 4-cycles. 
and get that both $y_{1}$ and $y_{k}$ are in clauses with both $x$ and $\bar{x}$, so they must be false by (7), but then $z_{2}=x$, so $y_{i}$ must also be false by (7). Now, both $z_{3}$ and $z_{4}$ must be equal to $x$, but then $x$ must be false by (5) and $y_{j}$ must also be false. This is a contradiction: since none of the $y$ 's are the same by Lemma 12 , at least two of them are from the same clause with $x$, but they are all false and so is $x$.

Suppose that none of the $z$ 's is negated. If an odd number of the $y$ 's are negated we reduce $F$ by $(18)$ and if two $y$ 's in neighbouring clauses are negated, we reduce $F$ by Lemma 13 . If two $y$ 's in "opposite" clauses on the cycle are negated, $x$ must be false: if $x$ is true, all $y$ 's are set to false and by the two clauses with negated $y$ 's all the $z$ 's are set to false, but then the other two $y$ 's must be true, a contradiction, so $x$ must be false. In the remaining cases, none of the y's is negated. If two $y$ 's in neighbouring clauses are from the same clause with $x$ we have the cycle in Figure $14(\mathrm{~b})$, but then $z_{2}=z_{4}$ : suppose $z_{2}=\bar{z}_{4}$; then $z_{1}$ and $z_{3}$ must be false by (7), but then $y_{1}=y_{2}=\bar{z}_{2}=z_{4}=\bar{y}_{3}$. Then $y_{1}$ and $y_{2}$ must be false, but then both $x$ and $y_{3}$ must be true, a contradiction.

Lemma 14. If a reduced formula $F$ contains a 4-cycle as in Figure 14(c), $F$ is satisfiable iff $F$ with the last clause on the cycle removed is satisfiable.

Proof. It is trivially true, that if $F$ is satisfiable, so is $F$ with the last clause removed. Suppose that $F$ without the last clause is satisfied. If $x$ is true, all the $y$ 's are false, so from the other three clauses, we have that $z_{1}=\bar{z}_{2}=z_{3}=\bar{z}_{4}$ so the fourth clause is satisfied. If $x$ is false, $y_{1}=\bar{y}_{2}$, so exactly one of the $z$ 's are true; if it is one of $z_{1}$ or $z_{4}, y_{3}$ must be true by the second clause so $y_{4}$ must be false and if it is one of $z_{2}$ or $z_{3}, y_{3}$ must be false and hence $y_{4}$ must be true by their common clause with $x$. In both cases, the last clause is satisfied.

The only remaining 4-cycles have no negations and no $y$ occurring more than once. Then at least two of the $y$ 's must be from the same clause with $x$ and they are not in neighbour clauses on the cycle. If the other two $y$ 's are also from the same clause with $x$, we transform $F$ by removing the last clause on the cycle by Lemma 14. If they are not from the same clause with $x$ the 4-cycle must look like the one in Figure 14(c), except $y_{4}$ is replaced by $y_{5}$. Then we add the clause $\left(y_{4}, z_{4}, z_{1}\right)$ by Lemma 14 and get that $y_{4}=y_{5}$ by $(14)$. 
F contains a clause with a negated variable from $Y$. There must be at least four clauses with variables from $Y$ other than the ones with $x$. We branch on $x$; setting it to false removes four variables and setting it to true sets all the $y$ 's to false and in each of the other clauses with $y$ 's either sets one of the $z$ 's to the negation of the other or both to false. This removes at least twelve variables, as at least one $y$ is negated and the $z$ 's do not form a 3- or 4-cycle.

5-cycles. If $F$ contains a 5-cycle as in Figure 12(c), none of the $y$ 's is negated by the previous case; also, none of the $y$ 's are the same by Lemma 12, so there must be four clauses on the 5-cycle, as in Figure 15 , that contain only $y$ 's from two clauses with $x$. The $y$ 's will always satisfy

$$
\begin{array}{r}
\left(x, y_{1}, y_{2}\right)\left(y_{1}, z_{1}, z_{2}\right) \text { An even number of the } z \text { 's are negated }: z_{1}=z_{5} \\
\left(x, y_{3}, y_{4}\right)\left(y_{i}, \tilde{z}_{2}, z_{3}\right) \text { An odd number of the } z \text { 's are negated }: z_{1}=\bar{z}_{5} \\
\left(x, y_{5}, y_{6}\right)\left(y_{j}, \tilde{z}_{3}, z_{4}\right) \\
\left(y_{k}, \tilde{z}_{4}, z_{5}\right)
\end{array}
$$

Figure 15: Four clauses from a 5-cycle.

an even number of these clauses: if $x$ is true, they satisfy zero and if $x$ is false they satisfy two, as they pairwise become each other's negation, since they were from only two different clauses with $x$. Now, let us look at $z_{2}, z_{3}$ and $z_{4}$. The negated ones will always satisfy exactly one of the clauses and the unnegated will either satisfy zero or two; thus, if the number of negated $z$ 's is odd, $z_{1}$ and $z_{5}$ must satisfy an odd number of the clauses for $F$ to be satisfiable, so $z_{1}=\bar{z}_{5}$ and if the number of negated $z$ 's is even, $z_{1}=z_{5}$ by the same argument. In both cases, we have reduced $F$.

F contains at least five clauses with $y$ 's and $z$ 's. Since the clauses do not form 3-, 4- or 5-cycles at this point, branching on $x$ yields at least $(12,4)$.

F contains exactly four clauses with y's and $z$ 's. As at least four variables from $Y$ occur in clauses without $x$ and no two variables from $Y$ occur together, there must be exactly four such clauses. Furthermore, two of the variables from $Y$ are unique, and the others are $(2,0)$-occurrences; otherwise, there would be more than four clauses with variables from $Y$. 
Since there are no cycles, we have already seen how to get $(11,4)$ branching on $x$. If the formula is $\frac{15}{2}$-sparse, we can solve the remaining formula in time $O\left(2^{\frac{2 n}{15}}\right)=O\left(2^{0.13333 n}\right)$. We want to branch on formulas with too many $(3,0)$-occurrences; we either prove that we remove an extra variable when branching on $x$ or we branch on a different variable.

Suppose $x_{1}$ is a $(3,0)$-occurrence occurring in the three first clauses in Figure 16. The other clause with $y_{3}$ contains no unique variable by (15). If it contains a variable $x_{2}$, that only occurs in other clauses with unique variables as in Figure 16, we branch on $x_{1}$; setting it to true removes

\begin{tabular}{|c|c|c|}
\hline $\begin{array}{l}\left(x_{1}, y_{1}, y_{2}\right) \\
\left(x_{1}, y_{3}, u_{1}\right) \\
\left(x_{1}, y_{5}, u_{2}\right)\end{array}$ & $\left(x_{2}, y_{3}, z\right)$ & $\begin{array}{l}\text { All other clauses with } x_{2} \\
\text { contain a unique variable. }\end{array}$ \\
\hline
\end{tabular}

Figure 16: Unique variables.

eleven variables and setting it to false removes $x_{1}, y_{2}, u_{1}$ and $u_{2}$, but then $y_{3}$ is unique, so all clauses with $x_{2}$ contain a unique variable and we set $x_{2}$ to false by (15). Then also $z=\bar{y}_{3}$ and we get a branching vector of at least $(11,6)$.

If another $(3,0)$-occurrence $x_{2}$ occurs with one of the variables from $Y$ it must be either $y_{1}$ or $y_{2}$ : suppose $x_{2}$ is in a clause with $y_{3}$. Now, $y_{3}$ is a $(2,0)$-occurrence with a unique variable in its clause with $x$, so its other clause can not contain unique variables; then the remaining clauses with $x_{2}$ must contain unique variables, but that is the previous case. Suppose $y_{1}$ is in a clause with $x_{2}$, then that clause can not contain a unique variable, as this would also be the previous case (by looking at $x_{2}$ instead of $x_{1}$ ). The two other clauses with $x_{2}$ must then contain a unique variable, so they do not contain $y_{3}$ or $y_{5}$ and if they contain $y_{2}$, we have a $(3,0)$-occurrence with two of the variables it occurs with occurring together in another clause, which is a previous case. So we must have the clauses in Figure 17 (to easier distinguish variables occurring with different $(3,0)$-occurrences, we use $y^{\prime}$ and $y^{\prime \prime}$ to denote variables occurring with other $(3,0)$-occurrences than $x_{1}$ in the rest of this section). Then we branch on $y_{2}$; setting it to true removes eight variables as shown in Figure 17 and setting it to false we get $z_{4}=\bar{z}_{3}$ and $y_{1}=\bar{x}_{1}$, as shown in the figure. Then we have $\bar{x}_{1}$ in a clause with $x_{2}$ and both of them only occur unnegated in clauses with unique variables elsewhere, so $x_{2}$ is set to false by (15), and we remove the remaining variables in the figure for a branching vector of at least $(8,7)$. 


$$
\begin{aligned}
& \left(x_{1}, y_{1}, y_{2}\right)\left(y_{1}, x_{2}, y_{2}^{\prime}\right)\left(x_{2}, y_{3}^{\prime}, u_{1}^{\prime}\right) y_{2}=\text { true }: x_{1}=y_{1}=z_{3}=z_{4}=\text { false, } \\
& \left(x_{1}, y_{3}, u_{1}\right)\left(y_{2}, z_{3}, z_{4}\right)\left(x_{2}, y_{5}^{\prime}, u_{2}^{\prime}\right) \quad u_{1}=\bar{y}_{3}, u_{2}=\bar{y}_{5}, y_{2}^{\prime}=\bar{x}_{2} \\
& \left(x_{1}, y_{5}, u_{2}\right)\left(y_{3}, z_{5}, z_{6}\right) \quad y_{2}=\text { false }: y_{1}=\bar{x}_{1}, z_{4}=\bar{z}_{3} \text {, } \\
& \left(y_{5}, z_{7}, z_{8}\right) \quad x_{2} \stackrel{15}{=} \text { false, } y_{2}^{\prime}=x_{1} \text {, } \\
& u_{1}^{\prime}=\bar{y}_{3}^{\prime}, u_{2}^{\prime}=\bar{y}_{5}^{\prime}
\end{aligned}
$$

Figure 17: One variable from $Y$ occurs with another $(3,0)$-occurrence.

Now, no variable from $Y$ occurs with another $(3,0)$-occurrence. Suppose both $z_{5}$ and $z_{6}$ occur with a $(3,0)$-occurrence. Then $z_{5}$ and $z_{6}$ can not be negated, as a $(3,0)$-occurrence where one of the variables it occurs with occurs negated in another clause is a previous case; also, the clause with $z_{5}$ and $z_{6}$ contains $y_{3}$, which is only in one other clause and that clause contains a unique variable, so if the clause with $z_{5}$ (or $z_{6}$ ) and the $(3,0)$-occurrence contains a unique variable, we have the case in Figure 16 (with the $(3,0)$-occurrence as $x_{1}$ and $z_{5}$ as $y_{3}$ ). Now, $z_{5}$ and $z_{6}$ can not occur in another clause together, so we must have the clauses in Figure 18 . We branch on $z_{6}$; setting it to true removes nine variables

\begin{tabular}{|cccc|}
\hline$\left(x_{1}, y_{1}, y_{2}\right)$ & $\left(y_{1}, z_{1}, z_{2}\right)$ & $\left(x_{2}, z_{5}, y_{2}^{\prime}\right)$ & $\left(x_{3}, z_{6}, y_{2}^{\prime \prime}\right)$ \\
$\left(x_{1}, y_{3}, u_{1}\right)$ & $\left(y_{2}, z_{3}, z_{4}\right)$ & $\left(x_{2}, y_{3}^{\prime}, u_{1}^{\prime}\right)$ & $\left(x_{3}, y_{3}^{\prime \prime}, u_{1}^{\prime \prime}\right)$ \\
$\left(x_{1}, y_{5}, u_{2}\right)$ & $\left(y_{3}, z_{5}, z_{6}\right)$ & $\left(x_{2}, y_{5}^{\prime}, u_{2}^{\prime}\right)$ & $\left(x_{3}, y_{5}^{\prime \prime}, u_{2}^{\prime \prime}\right)$ \\
& $\left(y_{5}, z_{7}, z_{8}\right)$ \\
$z_{6}=$ true $: y_{3}=z_{5}=x_{3}=y_{2}^{\prime \prime}=$ false, $u_{1}^{\prime \prime}=\bar{y}_{3}^{\prime \prime}, u_{2}^{\prime \prime}=\bar{y}_{5}^{\prime \prime}, u_{1}=\bar{x}_{1}, y_{2}^{\prime}=\bar{x}_{2}$ \\
$z_{6}=$ false $: y_{2}^{\prime \prime}=\bar{x}_{3}, z_{5}=\bar{y}_{3}, x_{2} \stackrel{15}{=}$ false $, y_{2}^{\prime}=y_{3}, u_{1}^{\prime}=\bar{y}_{3}^{\prime}, u_{2}^{\prime}=\bar{y}_{5}^{\prime}$
\end{tabular}

Figure 18: Both $z_{5}$ and $z_{6}$ occur with a $(3,0)$-occurrence.

as shown in the figure, and setting $z_{6}$ to false sets $y_{2}^{\prime \prime}=\bar{x}_{3}$ and $z_{5}=\bar{y}_{3}$. Now, $x_{2}$ is in a clause with $\bar{y}_{3}$ and both variables only occur unnegated in clauses with unique variables elsewhere, so we set $x_{2}$ to false by (15) and remove $y_{2}^{\prime}, u_{1}^{\prime}$ and $u_{2}^{\prime}$. In total, we get a branching vector of at least $(9,7)$.

Sparse formulas. Now, no $y$ can occur with another $(3,0)$-occurrence, so there are at least six variables occurring at most twice for each $(3,0)$ occurrence. Also, as $z_{5}$ and $z_{6}$ (and by symmetry $z_{7}$ and $z_{8}$ ) do not both occur with a $(3,0)$-occurrence, we can assume that neither $z_{5}$ nor $z_{7}$ occur 
with a $(3,0)$-occurrence. As they both occur at most twice, they are in clauses with at most four different variables; thus, we count each of them as one fourth of a variable for each of the $(3,0)$-occurrences, whose $y$ they occur with. This means, that there are at least six and a half variables occurring at most twice for each $(3,0)$-occurrence; thus, the formula is $\frac{15}{2}$-sparse and we solve it in time $O\left(2^{\frac{2 n}{15}}\right)=O\left(2^{0.13333 n}\right)$.

\section{Conclusion}

The main result of this paper is the following theorem.

Theorem 1. The presented algorithms for XSAT and X3SAT run in time $O\left(2^{0.2325 n}\right)$ and $O\left(2^{0.1379 n}\right)$, respectively.

Proof. When our algorithms are applied to a formula $F$ with $m$ clauses and $n$ variables, the sizes of the intermediate formulas are never larger than $\max (|F|, 2 m n)$ : they are never larger during the reduction procedures by Lemma 3 and after the reduction procedures have run, the formula has size at most $m n$. In some of the branches we add a clause, but when we call the algorithm recursively, the reduction procedure will remove a clause, so the size is never larger than $\max (|F|, 2 m n)$, which is polynomial in the size of the original formula. Also, no reduction or branching adds variables. The number of recursive calls are at most $O\left(2^{0.2325 n}\right)$ and $O\left(2^{0.1379 n}\right)$, respectively, by Section 3.2 and Section 3.3 . For each recursive call, the reduction procedure runs in polynomial time in the size of the formula by Lemma 4 and we can in polynomial time decide, which case to branch on. Since we ignore the polynomial factors, we get the stated running times.

Both our algorithms are extensions of known branch-and-reduce algorithms. One important addition to the algorithms are new reductions, which limit the number of possible structures of the formula. The other important addition is the concept of sparse formulas, which in certain situations enables us to simply enumerate all possible assignments to the variables we would otherwise branch on and leaves us with a problem that is solvable in polynomial time. One could hope that the concept of sparse formulas is also useful in other algorithms. 


\section{Acknowledgements}

We would like to thank the anonymous referees for many helpful comments. We are also grateful to our supervisors Peter Bro Miltersen and Sven Skyum for their help with this paper.

\section{References}

[1] L. Drori and D. Peleg. Faster exact solutions for some NP-hard problems. Theoretical Comput. Sci., 287(2):473-499, 2002.

[2] A. S. Kulikov. An upper bound $O\left(2^{0.16254 n}\right)$ for exact 3-satisfiability: A simpler proof. Zapiski nauchnyh seminarov POMI, 293:118-128, 2002. Can be found at http://logic.pdmi.ras.ru/ kulikov/ x3sat_e.ps.gz.

[3] O. Kullmann and H. Luckhardt. Deciding propositional tautologies: Algorithms and their complexity, 1997. Manuscript. Can be found at http://cs-svr1.swan.ac.uk/ csoliver/tg.ps.gz.

[4] B. Monien, E. Speckenmeyer and O. Vornberger. Upper bounds for covering problems. Bericht 7, Universität Paderborn, 1980.

[5] B. Monien, E. Speckenmeyer and O. Vornberger. Upper bounds for covering problems. Methods of Operations Research, 43:419-431, 1981.

[6] S. Porschen, B. Randerath and E. Speckenmeyer. Exact 3-satisfiability is decidable in time $O\left(2^{0.16254}\right)$, 2002. To appear in Annals of Mathematics and Artificial Intelligence.

[7] S. Porschen, B. Randerath and E. Speckenmeyer. X3SAT is decidable in time $O\left(2^{n / 5}\right), 2002$. Manuscript. Can be found at http://gauss . ececs.uc.edu/Conferences/SAT2002/Abstracts/ porschen.ps

[8] T. J. Schaefer. The complexity of satisfiability problems. In Proceedings of the Tenth Annual ACM Symposium on Theory of Computing, pages 216-226, 1978.

[9] R. Schroeppel and A. Shamir. A $T=O\left(2^{n / 2}\right), S=O\left(2^{n / 4}\right)$ algorithm for certain NP-complete problems. SIAM J. Comput., 10(3):456-464, 1981. 


\section{Recent BRICS Report Series Publications}

RS-03-30 Jesper Makholm Byskov, Bolette Ammitzbøll Madsen, and Bjarke Skjernaa. New Algorithms for Exact Satisfiability. October 2003. 31 pp.

RS-03-29 Aske Simon Christensen, Christian Kirkegaard, and Anders Møller. A Runtime System for XML Transformations in Java. October 2003. 15 pp.

RS-03-28 Zoltán Ésik and Kim G. Larsen. Regular Languages Definable by Lindström Quantifiers. August 2003. 82 pp. This report supersedes the earlier BRICS report RS-02-20.

RS-03-27 Luca Aceto, Willem Jan Fokkink, Rob J. van Glabbeek, and Anna Ingólfsdóttir. Nested Semantics over Finite Trees are Equationally Hard. August 2003. 31 pp.

RS-03-26 Olivier Danvy and Ulrik P. Schultz. Lambda-Lifting in Quadratic Time. August 2003. 23 pp. Extended version of a paper appearing in Hu and Rodríguez-Artalejo, editors, Sixth International Symposium on Functional and Logic Programming, FLOPS '02 Proceedings, LNCS 2441, 2002, pages 134-151. This report supersedes the earlier BRICS report RS-02-30.

RS-03-25 Biernacki Dariusz and Danvy Olivier. From Interpreter to Logic Engine: A Functional Derivation. June 2003.

RS-03-24 Mads Sig Ager, Olivier Danvy, and Jan Midtgaard. A Functional Correspondence between Call-by-Need Evaluators and Lazy Abstract Machines. June 2003. 13 pp.

RS-03-23 Korovin Margarita. Recent Advances in $\Sigma$-Definability over Continuous Data Types. June 2003. 26 pp.

RS-03-22 Ivan B. Damgård and Mads J. Jurik. Scalable Key-Escrow. May 2003. 15 pp.

RS-03-21 Ulrich Kohlenbach. Some Logical Metatheorems with Applications in Functional Analysis. May 2003. 55 pp. Slighly revised and extended version to appear in Transactions of the American Mathematical Society. 